\title{
La représentation des surfaces continentales dans la modélisation du climat à Météo-France
}

Bertrand Decharme $^{(1)}$, Christine Delire ${ }^{(1)}$, Aaron Boone ${ }^{(1)}$

Corresponding Author: Bertrand Decharme

Météo-France, CNRM/GMGEC/EST

42 av. G. Coriolis, 31057 Toulouse, France

E-Mail: bertrand.decharme@ meteo.fr

(1) Centre national de recherches météorologiques, UMR 3589, Météo-France/CNRS, Université de Toulouse, France 


\section{Résumé}

Les surfaces continentales jouent un rôle non-négligeable dans le système climatique de la Terre. Elles occupent d'ailleurs une place majeure dans les cycles globaux de l'eau et du carbone. Elles ont été prises en compte dès les premiers modèles numériques de climat et, avec l'évolution des connaissances, des capacités de calcul et de la demande sociétale, leur représentation s'est aujourd'hui considérablement complexifiée. Nous présentons ici une brève histoire de l'évolution du modèle de surfaces Isba (Interactions Sol-BiosphèreAtmosphère) de Météo-France dans son utilisation à l'échelle du globe en la replaçant dans le contexte international de la modélisation climatique.

\section{Abstract}

Land surfaces play a significant role in the Earth climate system, and they are a major component of the global carbon and water cycles. The first numerical climate models took them into account in very simple ways. Through time the complexity of their representation has increased a lot owing to improved knowledge, larger computational resources and changing societal demands. We present here a brief history of the Isba (Interactions SoilBiosphere-Atmosphere) land surface model developed at Météo-France when used at global scale and how it evolved in the context of international climate modelling. 


\section{(Introduction)}

Le système climatique de la Terre décrit l'ensemble des interactions entre l'atmosphère, les océans et les continents qui, principalement sous l'effet du rayonnement solaire, détermine le climat de la planète. Ces interactions sont liées aux échanges d'énergie, de quantité de mouvement et de matière entre ces trois entités. Les échanges de matières qui conditionnent le plus l'évolution de ce système sont principalement régis par les cycles de l'eau et du carbone. Ces deux cycles sont d'ailleurs intimement liés et en forte interaction avec le bilan d'énergie à la surface de la Terre. Des perturbations externes d'origine naturelles ou anthropiques peuvent aussi fortement influencer l'évolution de ce système à plus ou moins long terme. Les éruptions volcaniques par exemple émettent des particules sulfatées dans la haute atmosphère entrainant une augmentation de la part du rayonnement solaire réémis vers l'espace et donc un refroidissement de la planète. A l'inverse, l'utilisation de combustibles fossiles par l'homme engendre une augmentation de la teneur en dioxyde de carbone dans l'atmosphère créant un déséquilibre dans l'effet de serre de la Terre qui réchauffe tant la surface que la couche atmosphérique proche d'elle. C'est d'ailleurs ce dernier processus qui explique les réchauffements climatiques actuels (Planton et al., 2015).

Bien que la variabilité naturelle du système climatique soit surtout pilotée par l'atmosphère et l'océan, les continents sont de plus en plus considérés comme un acteur important de ce système. Outre les contrastes Terre-mer qui engendrent des gradients thermiques et des variations dans le champ de pression terrestre, les caractéristiques physiques des surfaces continentales modulent aussi les échanges de quantité de mouvement avec l'atmosphère via les frottements induits par la présence de montagnes et de végétation sur la fine couche limite atmosphérique qui la surplombe. Les surfaces continentales sont aussi un lieu d'échanges privilégiés de matière et d'énergie. De nombreux processus sont à l'œuvre, de l'échelle de la plante à l'échelle planétaire, avec une complexité et une hétérogénéité bien plus importante qu'ailleurs. La présence d'humidité dans les sols, de végétation, de neige, de glace, et d'eau libre en surface joue un rôle majeur dans la régulation de ces échanges. Les fortes hétérogénéités des propriétés radiatives (albédo, émissivité) de ces différentes surfaces vont grandement moduler le bilan d'énergie de la Terre. Les échanges d'eau et de carbone sont fortement contraints par la diversité des paysages, de la topographie et des propriétés des sols présentes sur les continents. Enfin, la biosphère continentale est un acteur déterminant du système climatique puisque, couplée aux cycles de l'énergie et de l'eau, elle pilote la partie 
continentale du cycle du carbone et elle peut donc avoir un impact direct sur l'effet de serre planétaire.

Afin d'étudier ce système climatique, des modèles de climat couvrant l'ensemble du globe ont été développés en se concentrant sur la physique et la dynamique de l'atmosphère, des océans et de la glace de mer, et sur l'hydrométéorologie des surfaces continentales. Cette dernière est représentée par des modèles de surface qui avaient été développés à l'origine pour fournir des rétroactions « réalistes » aux modèles atmosphériques en termes de flux d'énergie, d'humidité et de quantité de mouvement. Récemment des modèles climatiques de nouvelle génération dits du «système Terre » ont été mis en place, principalement pour étudier le changement climatique et ses effets, en intégrant aux modèles de climat des représentations de l'évolution des aérosols, de la chimie atmosphérique, des écosystèmes continentaux et océaniques, et des cycles biogéochimiques tel celui du carbone ou de l'azote. Un des buts des modèles de climat et du « système Terre » est aussi la réalisation de scénarios climatiques des 20-21ème siècles demandés par le projet international Cmip (Coupled Model Intercomparison Project) du WCRP (World Climate Research Program) qui a pour but d'intercomparer les modèles de climat (Boucher et al., 2019). Ces simulations servent ensuite à établir les divers rapports sur l'évolution et l'impact du changement climatique édités par le Giec (Groupe d'experts Intergouvernemental sur l'Evolution du Climat; Jouzel et al., 2018).

\section{Les premières représentations de l'hydrométéorologie continentale}

Il y a 50 ans, un des pionniers de la modélisation climatique, Manabe (1969) choisit pour décrire l'hydrométéorologie des surfaces continentales l'approche dite «baquet » ou plus communément «seau d'eau » où le sol est représenté comme un seul réservoir globalement uniforme de $1 \mathrm{~m}$ de profondeur simulant l'évaporation réelle comme une simple fraction de l'évaporation potentielle (cf. Beljaars et Mahfouf, 2020). Par la suite, Bhumralkar (1975) proposa une méthode simple, dite «force-restore », afin de résoudre le cycle diurne de la température à la surface et dans le sol. La tendance temporelle de la température à la surface du sol était forcée par le bilan d'énergie (rayonnement net moins la somme des chaleurs sensible et latente) et rappelée vers sa propre moyenne journalière, représentant ainsi une pseudo-température du sol. Ces approches «seau d'eau» pour l'hydrologie et «forcerestore » pour la thermique dans les sols furent d'ailleurs utilisées dans les années 90 pour 
représenter les surfaces continentales dans la première version d'Arpege ${ }^{1}$-Climat, le modèle atmosphérique de Météo-France toujours utilisé aujourd'hui pour des applications climatiques globales. Cette modélisation très simple présentait l'avantage de limiter le nombre de paramètres à calibrer et de se passer du traitement de l'équation de diffusion de la chaleur décrite par la loi de Fourier et de diffusion de l'eau décrite par l'équation de Richards dérivée de la loi de Darcy pour décrire le transport de l'énergie et de l'humidité dans le sol diminuant ainsi le coût numérique des simulations.

Néanmoins, bien que cette modélisation permît de résoudre le cycle diurne de la température proche de la surface, il n'en était pas de même pour l'humidité superficielle du sol qui pourtant impacte fortement le bilan d'énergie en surface (et donc la température) via son contrôle sur l'évaporation du sol nu. Ce problème fut résolu par Deardorff (1977) qui généralisa la méthode «force-restore » au transport vertical de l'eau dans le sol dans un modèle de type «seau d'eau ». Il rajouta une fine couche de surface incluse dans le réservoir de sol afin de mieux décrire l'évolution temporelle de l'humidité superficielle. Mais qu'en est-il de la végétation qui recouvre la majorité des continents ? Deardorff (1978) fut alors le premier à proposer une paramétrisation simple incluant une couche de végétation pour le calcul des bilans d'eau et d'énergie. Cette approche représentait la transpiration des plantes et l'évaporation du feuillage suite à l'interception de la pluie par la canopée. Bien des travaux furent menés dans les années 70 sur la représentation des surfaces continentales. Parmi ceuxci on peut citer le travail de Jarvis (1976) qui permit de représenter plus ou moins simplement le rôle des stomates qui régulent la transpiration des plantes par une conductance stomatique (l'inverse de la résistance) empiriquement reliée aux conditions environnementales qui contrôlent la photosynthèse (rayonnement, température, déficit de pression de vapeur saturante, stress hydrique). Cette conductance stomatique était ensuite multipliée par un indice de surface foliaire climatologique pour représenter l'ensemble de la canopée. A l'orée des années 80 , tous ces travaux donnèrent naissance aux premiers modèles de surface, comme BATS (Biosphere Atmosphere Transfer Scheme; Dickinson et al. 1986) ou SiB (Simple Biosphere model; Sellers et al. 1986), qui bien que simulant simplement tous les processus physiques décrits plus avant, présentaient le désavantage de modéliser les plantes de manière très détaillée utilisant un nombre très important de paramètres biophysiques pour la plupart

\footnotetext{
${ }^{1}$ Action de recherche petite échelle grande échelle
} 
non mesurés afin de simuler des bilans d'énergie séparés pour le sol et la végétation (cf Beljaars et Mahfouf, 2020).

A l'inverse, comme détaillé dans Planton et Mahfouf (2020), Joël Noilhan fit à cette époque au CNRM le choix de la simplicité en proposant le modèle de surface Isba (Noilhan et Planton, 1989) qui reposait sur un nombre limité de paramètres et sur la méthode force-restore pour calculer un bilan d'énergie composite neige-sol-végétation à l'interface surfaceatmosphère. Son hydrologie était de type « seau d'eau » où le sol était décrit par une pseudocouche superficielle très mince en surface incluse dans une zone racinaire plus profonde. A la base de ce sol, Isba représentait le drainage gravitationnel de l'eau (voir Planton et Mahfouf, 2020) ce qui en faisait un modèle plus réaliste que le simple «seau d'eau » de Manabe (1969). La végétation recueillait la pluie interceptée par la canopée dans un réservoir dont une partie était évaporée et une autre s'égouttait jusqu'au sol. Joël Noilhan fut aussi l'un des tout premiers à proposer que la transpiration des plantes et donc la conductance stomatique soient représentées suivant l'approche de Jarvis (1976) sans prendre en compte la photosynthèse. La couverture neigeuse étant aussi une composante importante du système climatique, une représentation simple de la neige fut ajoutée dans Isba durant la thèse d'Hervé Douville via un schéma monocouche resté fidèle à la méthode «force-restore » qui décrivait l'évolution de sa densité et de son albédo en fonction du temps qui passe (Douville et al., 1995). Au milieu des années 90 cette version d'Isba (Figure 1a) fut introduite dans Arpege-Climat (Mahfouf et al., 1995) et à l'orée des années 2000 cette configuration fut couplée au modèle océanique Nemo (Nucleus for European Modelling of the Ocean) et au modèle de glace de mer Gelato (Global Experimental Leads and ice for ATmosphere and Ocean) pour donner naissance à CNRMCM2 (CNRM Coupled Model version 2), la deuxième version du modèle de climat développé conjointement par le CNRM et le Cerfacs (Centre Européen de Recherche et de Formation Avancée en Calcul Scientifique). Notons que dans cette version, aucune modélisation des rivières n'était couplée à Isba (figure 1a) et des climatologies d'eau douce étaient alors prescrites au modèle océanique Nemo.

Dans le même temps, les modèles de surface utilisés à l'échelle globale ont été découplés des modèles climatiques et utilisés en mode «forcé » pour mieux les intercomparer dans le cadre de projets internationaux ayant pour ambition d'améliorer ces modèles. Ainsi, les mêmes variables météorologiques issues de réanalyses ou d'observations (donc non affectées des biais systématiques des modèles atmosphériques) ont pu être imposées en entrée de tous les modèles de surface existant dans le monde. Les premières implications du CNRM, 
et en particulier de Joël Noilhan, sont décrites dans Planton et Mahfouf (2020). Ces projets ont montré, par exemple, que la plupart des divergences entre modèles étaient dues à la partition des pluies entre évapotranspiration et ruissellement pointant $\mathrm{du}$ doigt la représentation du ruissellement de surface dans les modèles. Fort de cette conclusion, une représentation empirique du ruissellement sur surface saturée pour des études climatiques ou hydrologiques fut introduite dans Isba durant la thèse de Florence Habets dirigée par Joël Noilhan. Cette représentation était basée sur une approche simple où la fraction du sol saturé en eau au sein de la maille était déterminée en fonction de l'humidité moyenne du sol, de l'intensité des précipitations et d'un paramètre empirique à calibrer (Habets et al., 1999). L'infiltration dans le sol était alors donnée par la différence entre ce ruissellement de surface et l'eau arrivant sur le sol, cette dernière étant la somme des précipitations non interceptées par la canopée, de l'égouttement de l'eau interceptée par la végétation et de la fonte des neiges (figure 1b). C'est d'ailleurs à partir de ce moment-là qu'Isba sera systématiquement utilisé en mode «forcé» à l'échelle globale que ce soit pour des applications hydrologiques ou pour valider des changements dans sa physique avant de les introduire dans notre modèle de climat.

\section{Vers une modélisation plus réaliste}

L'étude du climat requiert que les cycles de l'eau soient fermés à l'échelle globale et donc que les modèles de surface soient couplés à des modèles de routage de l'eau en rivière qui permettent de convertir le ruissellement simulé en débits pour transporter l'eau continentale vers les océans. Ces modèles de rivière facilitent aussi la validation des bilans d'eau calculés sur de vastes bassins hydrologiques via la comparaison des débits simulés et observés (Figure 2). Le modèle global de rivières Trip (Total Runoff Integrating Pathways) développé à l'université de Tokyo avec un réseau hydrographique de $1^{\circ}$ de résolution $(\sim 100 \mathrm{~km})$ et une vitesse d'écoulement uniforme fixée à $0.5 \mathrm{~m} . \mathrm{s}^{-1}$ fut alors couplé à ISBA sur tous les fleuves du monde afin d'en simuler les débits (Figure 1b). Notons aussi que pour les besoins de la prévision opérationnelle du temps, Isba fut modifié pour tenir compte du gel de l'eau des sols dans la zone racinaire (Giard et Bazile, 2000). Outre son impact sur les températures du sol en hiver, ce processus contribue à stocker l'eau durant les saisons froides pour la libérer lors des saisons plus chaudes. Enfin, deux activités facilitèrent l'utilisation d'Isba à l'échelle globale tant en mode «forcé » que couplé à l'atmosphère : (1) le travail précurseur de Joël Noilhan au milieu des années 1990 qui permit d'établir des règles d'agrégation spatiale (voir Planton et Mahfouf, 2020) pour les paramètres de sol et de 
végétation (Noilhan et Lacarrère, 1995); et (2) le développement au début des années 2000 d'une base de données physiographiques nommée Ecoclimap (Ecosystem Climate Maps) qui donna accès à la cartographie de ces paramètres à $1 \mathrm{~km}$ de résolution sur tous les continents. Aux milieux des années 2000, Isba-Trip et Ecoclimap furent intégrés à la troisième version du modèle de climat du CNRM-Cerfacs, CNRM-CM3 (http://www.umrcnrm.fr/scenario2004/references_eng.html), devenant la première version de CNRM-CM à fermer le cycle de l'eau à l'échelle globale. Ce modèle participa à Cmip3, la troisième phase du projet Cmip et contribua à l'élaboration du quatrième rapport du Giec.

Néanmoins, suite aux limites montrées par cette version d'Isba utilisée dans CNRM$\mathrm{CM} 3$, une couche de sub-surface de $50 \mathrm{~cm}$ à $1 \mathrm{~m}$ d'épaisseur fut rajoutée sous la couche racinaire durant la thèse d'Aaron Boone (Boone, 2000) dirigée par Joël Noilhan. Elle a permis de limiter l'asséchement estival trop précoce de la couche racinaire en autorisant des échanges d'eau par capillarité avec cette zone plus humide. Dans cette version à trois couches (figure 1c), le gel du sol fut aussi amélioré pour permettre de dissocier le gel superficiel en surface du gel plus profond dans la zone racinaire. De plus, une conclusion générale de plusieurs projets internationaux d'inter-comparaison auxquels le CNRM continuait de participer fut que la variabilité dite «sous-maille» de l'humidité des sols, due aux hétérogénéités des propriétés de sol et à la distribution des pluies, de la végétation ou de la topographie à l'intérieur d'une même maille, a un impact significatif sur le ruissellement, l'infiltration et donc le bilan d'eau générés par les modèles de surface. Un jeu de paramétrisations hydrologiques sous-mailles fut donc introduit dans Isba durant la thèse de Bertrand Decharme codirigée par Hervé Douville et Joël Noilhan. Il s'agissait de représenter : (1) le ruissellement sur une surface saturée à partir d'une représentation «statistico-dynamique» de la répartition observée de la topographie au sein de la maille ; (2) l'infiltration via deux distributions exponentielles sousmailles de l'intensité des précipitations et de la capacité maximale d'infiltration des sols tenant compte du gel ; et (3) l'hétérogénéité de la végétation via une approche type «mosaïque ${ }^{1}$. Ce jeu de paramétrisations sous-mailles a eu pour effet de favoriser le ruissellement total au détriment de l'évapotranspiration continentale améliorant ainsi le cumul annuel et, dans une moindre mesure, la dynamique saisonnière de la simulation des débits des grands fleuves du monde comme cela peut se voir sur la figure 2. L'augmentation de l'amplitude du pic saisonnier de crue des fleuves arctiques est principalement due au ruissellement sur sol gelé

\footnotetext{
${ }^{1}$ Calcul des bilans d'eau et d'énergie distincts pour chaque type de surface présent au sein de la maille qui sont ensuite moyennés en tenant compte de la fraction de chacun de ces types
} 
représenté dans cette version dite « $\mathrm{cm} 5$ » alors qu'ailleurs c'est surtout la prise en compte de la topographie dans le calcul du ruissellement de surface et, dans une moindre mesure, de l'hétérogénéité de la végétation qui tendent à augmenter les débits comparés à la version précédente dite « $\mathrm{cm} 3$ ». A la fin des années 2000, au regard de la qualité des débits simulés, cette modélisation permettait alors à ISBA de résider en bonne place dans la hiérarchie des modèles de surface utilisés pour des applications climatiques à l'échelle globale (Decharme and Douville, 2007). Grâce à l'avènement de la plateforme de modélisation Surfex ${ }^{1}$ (Le Moigne et Minvielle, 2019), cette configuration d'Isba couplée à Trip (figure 1c) fut introduite dans le modèle CNRM-CM5, la cinquième version du modèle de climat du CNRMCerfacs (https://www.umr-cnrm.fr/spip.php?article126). Ce modèle participa entre autres à l'exercice Cmip5 et contribua donc à l'élaboration du cinquième rapport du Giec.

\section{Aujourd'hui, une représentation plus ambitieuse}

A la fin des années 2000, l'avènement de nouvelles observations spatiales croisées avec l'augmentation des bases de données de mesures in-situ des débits sur l'ensemble du globe ont permis de montrer que cette version CNRM-CM5 d'Isba-Trip (https://www.umrcnrm.fr/spip.php?article131) était limitée par sa trop grande simplicité. Des erreurs importantes persistaient dans la modélisation du bilan d'eau continental, comme le souligne la mauvaise simulation du cycle annuel moyen des débits des grands fleuves du monde (figure 2). Aux échelles de temps annuelles et interannuelles, une première source d'erreurs était liée à une sous-estimation de l'évaporation continentale qui peut se déduire de la surestimation des débits sur quelques bassins arctiques et sur la plupart des bassins tropicaux. Ce défaut s'expliquait en partie par la non représentation de l'évaporation potentielle des vastes plaines d'inondations saisonnières qui apparaissent chaque année dans ces régions. Aux échelles de temps mensuelles à saisonnières, des erreurs systématiques de phase et d'amplitude dans les simulations des débits et du stock d'eau continental pouvaient être attribuées à la simplicité de Trip. Premièrement, les erreurs de modélisation dues à la vitesse d'écoulement de l'eau prise constante dans Trip étaient potentiellement importantes sur la plupart des bassins du monde et tout spécialement sur les fleuves arctiques comme le mauvais phasage des pics de crues le montre la figure 2. Or dans ces régions, la vitesse de l'eau varie fortement en quelques jours après la fonte des neiges dépassant très facilement la valeur de $0.5 \mathrm{~m} . \mathrm{s}^{-1}$. Deuxièmement,

\footnotetext{
${ }^{1}$ Surface externalisée
} 
l'absence de représentation des nappes aquifères, qui stockent une large part de l'eau infiltrée durant les saisons humides a restituant aux rivières durant les saisons plus sèches pour soutenir les débits d'étiages, était fortement préjudiciable.

De plus, la version type «seau d'eau » d'Isba ne pouvait pas modéliser précisément le transport de l'eau et de l'énergie à travers le manteau neigeux et chaque horizon du sol et donc les changements de phase eau/glace associés. Elle était en outre incapable de représenter les régimes hydrologique et thermique du pergélisol, comme l'épaisseur de sa couche active dégelant chaque été. Ainsi, la forte contribution aux débits des rivières boréales venant du ruissellement de la fonte de la neige à la surface du sol avant le dégel en profondeur ne pouvait pas être explicitement décrite. Enfin, le pergélisol est aussi un acteur potentiellement clef pour l'étude de l'évolution des changements climatiques car il contient une grande partie du stock de carbone terrestre. Sa fonte et la dégradation de cette matière organique via l'activité bactérienne dans le sol pourraient entrainer un accroissement significatif des émissions de dioxyde de carbone $\left(\mathrm{CO}_{2}\right)$ et de méthane $\left(\mathrm{CH}_{4}\right)$ vers l'atmosphère.

Au cours des années 2010, grâce à l'augmentation des moyens de calcul, le développement d'une modélisation hydrométéorologique plus ambitieuse des continents a donc été entreprise (Figure 3) en vue de la contribution de Météo-France au sixième rapport du GIEC et de la participation à CMIP6 de la nouvelle génération de nos modèles de climat CNRM-CM6 et du « système Terre » CNRM-ESM2 (http://www.umr-cnrm.fr/cmip6/). Cette nouvelle modélisation (cf. Decharme et al., 2019) repose en partie sur l'amélioration d'une autre facette du travail de thèse d'Aaron Boone dirigée par Joël Noilhan qui, au début des années 2000, consista à modéliser de manière explicite les transferts d'énergie et d'humidité dans le sol et la neige. Dans cette nouvelle version et à l'image de nombreux modèles de surface d'aujourd'hui, Isba résout explicitement les équations de la chaleur et de l'eau dans un sol décrit par 14 couches d'épaisseurs croissantes de la surface jusqu'à $12 \mathrm{~m}$ de profondeur pour simuler la diffusion de l'énergie et de l'eau en tenant compte des propriétés thermiques et hydrauliques des parties minérales et organiques du sol. L'utilisation d'un schéma de neige multi-couches (12 couches) de complexité intermédiaire, un des plus complets utilisé aujourd'hui pour des applications climatiques globales, permet de simuler des bilans hydriques et énergétiques distincts pour le sol et le manteau neigeux. Ce schéma permet de bien mieux représenter la saisonnalité et l'amplitude de l'enneigement de l'hémisphère Nord comparé à l'ancien modèle comme le montre la Figure 4. Le fort couplage entre les températures de la neige et du sol est ainsi modélisé ce qui permet de simuler les pergélisols 
d'Amérique du Nord et de Sibérie (Figure 5). En effet, la Figure 5a montre que le modèle simule une limite du pergélisol en bon accord avec les estimations même s'il semble un peu trop s'étendre vers l'ouest en Sibérie. Les profondeurs des couches actives simulées et observées in-situ sur tout l'hémisphère Nord sont en très bon accord comme le souligne le faible biais moyen entre chaque mesure et le point de grille du modèle correspondant. Ce bon comportement général d'Isba est aussi confirmé sur la région de la Yakoutie (Figure 5b) où cette nouvelle version est comparée à des estimations spatialisées présentant un fort gradient latitudinal avec une augmentation de la profondeur de la couche active du nord au sud que le modèle est capable de reproduire.

Enfin, en termes d'hydrologie, le jeu de paramétrisations sous-mailles utilisé précédemment a été adapté à cette version. Le modèle de routage des fleuves a été complétement remanié et s'appelle aujourd'hui Ctrip, ce qui signifie «la version CNRM de Trip » pour le différencier d'autres versions existant dans le monde. L'ancien réseau de rivières à $1^{\circ}$ de résolution a été affiné à la résolution de $0.5^{\circ}$ et amélioré sur 1'Europe. La vitesse d'écoulement de l'eau en rivière est à présent résolue de manière dynamique en la reliant à la masse d'eau en rivière et en supposant une section transversale de rivière rectangulaire. Un véritable «aller-retour » entre Isba et Ctrip a été mis en place grâce à l'introduction dans Surfex d'une interface de couplage standardisée. Ce couplage permet l'utilisation d'un schéma d'inondations dynamiques des fleuves qui rend compte de l'interaction entre les plaines inondées, le sol et l'atmosphère via principalement l'évaporation et la ré-infiltration de l'eau libre en surface. La physique des nappes phréatiques et leurs couplages avec le sol superficiel sont aussi rendus possible grâce à un schéma d'aquifères diffusif bidimensionnel qui échange avec les rivières et à une paramétrisation sous-maille des flux capillaires vers le sol. Ce nouveau système appelé Isba-Ctrip (http://www.umrcnrm.fr/spip.php?article1092) démontre des performances bien supérieures à ses prédécesseurs pour, par exemple, simuler la saisonnalité et l'amplitude des débits des grands fleuves du monde comme le montre la Figure 2. Aujourd'hui, ce modèle est d'ailleurs le seul au monde à pouvoir simuler l'impact combiné des inondations saisonnières et des nappes phréatiques sur les bilans d'eau et d'énergie des surfaces continentales dans un modèle de climat. 


\section{Vers la modélisation du « système Terre »}

A la fin du $20^{\mathrm{e}}$ siècle, les préoccupations scientifiques et sociétales induites par le changement climatique ont fait apparaître le besoin d'intégrer dans les modèles climatiques de nouvelle génération dits du «système Terre » et, donc dans les modèles de surface, les processus biogéochimiques en lien avec le cycle du carbone (Friedlingstein et al., 2007). En effet, la biosphère continentale (la végétation et les sols) absorbe environ 30\% des émissions anthropiques de $\mathrm{CO}_{2}$. Des $39 \mathrm{GtCO}_{2}$ émises en moyenne par an sur 2008-2017 par l'activité humaine, seules 17.3 sont restées dans l'atmosphère. Le reste a été absorbé par la biosphère continentale $\left(12 \mathrm{GtCO}_{2}\right)$ et les océans $(9 \mathrm{Gt})$. La biosphère continentale régule donc en partie la concentration de $\mathrm{CO}_{2}$ atmosphérique, ce qui en fait un acteur essentiel pour comprendre l'évolution des changements climatiques actuels. De plus, l'efficacité de cette régulation dépend directement du climat. En cas de sécheresse comme en Amazonie en 2010 ou de vague de chaleur par exemple, l'absorption de $\mathrm{CO}_{2}$ diminue. Les progrès de la recherche en écophysiologie dans les années 70-80, surtout le modèle de photosynthèse de Farquhar et al. (1980) combiné à des modèles de conductance stomatique ont permis à partir des années 90 de représenter de manière mécanistique le couplage entre le cycle de l'eau et le cycle du carbone dans les modèles de surface. Bonan (1996) avec le modèle de surface du Ncar (National Center for Atmospheric Research), Sellers et al. (1996) avec SiB2 (Simple Biosphere model version 2) ou Foley et al. (1996) avec Ibis (Integrated BIosphere Simulator) sont les premiers à avoir inclus une représentation de la photosynthèse et du cycle du carbone dans la végétation des modèles de surface continentale couplés aux modèles de climat. Les premiers pas dans ce sens avec ISBA datent de la fin des années 90 quand la représentation empirique de la conductance stomatique de type Jarvis (1976) fut remplacée par des relations plus mécanistiques la reliant à l'assimilation du $\mathrm{CO}_{2}$ atmosphérique par la photosynthèse à l'échelle de la feuille (Calvet et al., 1998).

Depuis ces premiers travaux, Isba a beaucoup évolué et un schéma complet de la partie continentale du cycle du carbone (Figure 6) a été introduit dans Isba-Ctrip pour atteindre l'état de l'art requis par la modélisation du «système Terre » (Joetzjer et al., 2015 ; Seferian et al., 2019). En plus de l'assimilation du carbone par photosynthèse en bonne adéquation avec les observations (figure 7), Isba représente la respiration des plantes (appelée respiration autotrophe), l'évolution de leur biomasse, et de leur indice de surface foliaire qui peut aussi être comparé à des observations (figure 8). De plus, Isba tient compte des feux de 
forêt naturels de grande échelle qui constituent un facteur important de perturbation du fonctionnement de la biosphère et une source d'émission non négligeable de $\mathrm{CO}_{2}$. La fraction de superficie brûlée ainsi que les émissions de $\mathrm{CO}_{2}$ et de carbone suie associées sont ainsi modélisées dans Isba en chaque point de grille en fonction de la quantité de combustible disponible (la biomasse) et du degré d'humidité. Comme la quasi-totalité des autres modèles aujourd'hui, Isba simule aussi l'évolution du carbone du sol via l'approche Century (Parton et $a l .$, 1988) qui classe la matière organique du sol en réservoirs définis par leurs taux de décomposition présupposés. Les débris végétaux forment la litière aérienne (feuilles, tiges, bois) et sous-terraine (racines), qui est décomposée et incorporée aux réservoirs de carbone dans le sol. C'est la décomposition de cette matière organique par des micro-organismes (la respiration hétérotrophe) qui libère du $\mathrm{CO}_{2}$ vers l'atmosphère (figure 6) en fonction de la température et de l'humidité du sol. Elle ne se produit pas quand le sol est gelé, trop sec ou gorgé d'eau et sa représentation nécessite donc une bonne représentation des conditions hydriques et thermiques des sols. De plus, la percolation lente de l'eau à travers le sol engendre aussi la dissolution d'une partie du carbone solide qui y est contenu, c'est la lixiviation $^{1}$ des sols. Le carbone organique ainsi dissout va alors se retrouver dans les rivières pour y être transporté jusqu'à l'océan grâce à une simple équation de transport dans Ctrip dont la vitesse est équivalente à celle de l'écoulement de l'eau. Enfin, pour représenter l'évolution du climat aux $20^{\mathrm{e}}$ et $21^{\mathrm{e}}$ siècles, notre modèle du « système Terre » doit être équilibré dans un état climatique le plus stable possible, avant l'ère industrielle. Or l'utilisation des terres (déforestation, mise en culture, pâturages) a varié fortement depuis le $17^{\mathrm{e}}$ siècle, ce qui a modifié non seulement les échanges d'énergie et d'eau à la surface mais aussi les échanges de $\mathrm{CO}_{2}$. La représentation de ce changement de la distribution géographique des types de plantes dans Isba-Ctrip a donc aussi été introduit et permet aujourd'hui d'utiliser les cartes de changement d'utilisation des sols fournies, par exemple, pour les simulations du Giec.

\section{Conclusions}

Depuis le premier modèle de climat mis en place à Météo-France dans les années 1990, la représentation de la surface n'a eu de cesse d'évoluer jusqu'à nos jours sous l'impulsion notable de chercheurs comme Joel Noilhan ou Hervé Douville et, outre les auteurs de cet article, grâce au travail majeur réalisé par bon nombre de nos collègues sur le

\footnotetext{
${ }^{1}$ Dissolution dans l'eau des matières solides solubles, tel le carbone, contenues dans les sols
} 
modèle de surface Isba et la plateforme de modélisation Surfex. Les changements récents dans la modélisation de l'hydrométéorologie des surfaces continentales simulée par Isba-Ctrip ont représenté un effort significatif pour mettre à jour la physique de la neige et des sols, ainsi que pour prendre en compte les processus liés aux écoulements des rivières, aux nappes d'eau souterraines et aux inondations saisonnières. Tout ceci fait d'Isba-Ctrip un système hydrométéorologique de tout premier plan et en très bonne place dans l'état de l'art de la modélisation climatique globale. La représentation du cycle continental du carbone a été portée au niveau de l'état de l'art requis par la modélisation du «système Terre ». Elle est assez complète avec la prise en compte de l'utilisation des sols, des feux de forêts, la photosynthèse des plantes, la respiration du sol et de la végétation, la lixiviation du carbone présent dans la litière et dans les sols et son transport dans le réseau hydrographique jusqu'aux océans. Isba-Ctrip va ainsi permettre d'aborder des questions émergentes portant sur l'influence des réservoirs hydrologiques continentaux jusqu'ici non ou mal représentés dans les modèles (plaines inondées, aquifères, pergélisols) sur le climat, et vice-et-versa. Elle va aussi permettre d'étudier les liens entre le changement climatique, l'hydrologie et les émissions de carbone. In fine, elle devrait aussi faciliter les liens entre le CNRM et la communauté des impacts entre autres hydrologiques et écologiques. 


\section{Références}

Beljaars A., Mahfouf J.-F., 2020. Les premières représentations des surfaces continentales dans les modèles atmosphériques. La Météorologie, 108, xx-Xx

Bhumralkar C. M., 1975. Numerical experiments on the computation of ground surface temperature in an atmospheric general circulation model. J. Appl. Meteorol., 14(7), 12461258, doi:10.1175/1520-0450(1975)014<1246:neotco>2.0.co;2.

Bonan G. B., 1996. A land surface model (LSM version 1.0) for ecological, hydrological, and atmospheric studies: technical description and user's guide (No. NCAR/TN-417+STR)., Univ. Corp. Atmos. Res., doi:10.5065/D6DF6P5X.

Boone A., 2000. Modélisation des processus hydrologiques dans le schéma de surface Isba : inclusion d'un reservoir hydrologique, du gel et modélisation de la neige. Thèse de doctorat de l'Université Paul Sabatier - Toulouse III. https://www.umrcnrm.fr/IMG/pdf/boone_thesis_2000.pdf.

Boucher O., Denvil,S., Salas y Mélia, D., 2019. La participation des scientifiques français à CMIP6. La Météorologie, 105, 6-7, doi:10.4267/2042/70159.

Calvet J.-C., Noilhan J., Roujean J.-L., Bessemoulin P., Cabelguenne M., Olioso A. Wigneron J.-P., 1998. An interactive vegetation SVAT model tested against data from six contrasting sites. Agric. For. Meteorol., 92(2), 73-95, doi:10.1016/S0168-1923(98)00091-4.

Deardorff J. W., 1977: A parameterization of ground-surface moisture content for use in atmospheric models. J. Appl. Meteorol., 16(11), 1182-1185, doi:10.1175/15200450(1977)016<1182:APOGSM>2.0.CO;2.

Deardorff J. W., 1978. Efficient prediction of ground surface temperature and moisture, with inclusion of a layer of vegetation. J. Geophys. Res., 83(C4), 1889, doi:10.1029/JC083iC04p01889.

Decharme B., Douville H., 2007. Global validation of the ISBA sub-grid hydrology. Clim. Dyn., 29(1), doi:10.1007/s00382-006-0216-7.

Decharme B., Delire C., Minvielle M., Colin J., Vergnes J., Alias A., Saint-Martin D., Séférian R., Sénési S., Voldoire, A, 2019. Recent changes in the ISBA-CTRIP land surface system for use in the CNRM-CM6 climate model and in global off-line hydrological applications. J. Adv. Model. Earth Syst., 11(5), 2018MS001545, doi:10.1029/2018MS001545.

Dickinson R. E., Henderson-Sellers A., Kennedy J., Wilson F., 1986. Biosphere-atmosphere transfer scheme (BATS) for the NCAR community climate model, NCAR Technical Note NCAR/TN-275-+STR, Boulder, Colorado..

Douville H., Royer J.-F., Mahfouf J.-F., 1995. A new snow parameterization for the MétéoFrance climate model: Part I: validation in stand-alone experiments. Clim. Dyn., doi:10.1007/BF00208760.

Farquhar G. D., von Caemmerer S., Berry J. A., 1980. A biochemical model of photosynthetic $\mathrm{CO}_{2}$ assimilation in leaves of C3 species. Planta, 149(1), 78-90, doi:10.1007/BF00386231.

Foley J. A., Prentice I. C., Ramankutty N., Levis S., Pollard D., Sitch S., Haxeltine A., 1996. An integrated biosphere model of land surface processes, terrestrial carbon balance, and vegetation dynamics. Global Biogeochem. Cycles, 10(4), 603-628, doi:10.1029/96GB02692.

Friedlingstein P., Bopp L., Cadule, P., 2007. Changement climatique et cycle du carbone. La Météorologie, 58, 21-24, doi:10.4267/2042/18204. 
Giard D., Bazile E., 2000. Implementation of a wew assimilation scheme for soil and surface variables in a global NWP model. Mon. Weather Rev., 128, 997-1015, doi:10.1175/15200493(2000)128<0997:IOANAS>2.0.CO;2.

Habets F., Noilhan J., Golaz C., Goutorbe J.-P., Lacarrère P., Leblois E., Ledoux E., Martin E., Ottlé C., Vidal-Madjar D., 1999. The ISBA surface scheme in a macroscale hydrological model applied to the Hapex-Mobilhy area. Part I: Model and database. J. Hydrol., 217(1-2), 75-96, doi:10.1016/S0022-1694(99)00019-0.

Jarvis P. G., 1976. The interpretation of the variations in leaf water potential and stomatal conductance found in canopies in the field. Philos. Trans. R. Soc. B Biol. Sci., 273(927), $593-$ 610, doi:10.1098/rstb.1976.0035.

Joetzjer E., Delire C., Douville H., Ciais P., Decharme B., Carrer D., Verbeeck H., De Weirdt M., Bonal D., 2015. Improving the ISBACC land surface model simulation of water and carbon fluxes and stocks over the Amazon forest. Geosci. Model Dev., 8(6), 1709-1727, doi:10.5194/gmd-8-1709-2015.

Jouzel J., Petit M., Masson-Delmotte V., 2018. Trente ans d'histoire du Giec. La Météorologie,100, 117-124, doi:10.4267/2042/65154.

Mahfouf J.-F., Manzi A. O., Noilhan J., Giordani H., Déqué M., 1995. The land surface scheme ISBA within the Météo-France climate model ARPEGE. Part I: implementation and preliminary results. J. Clim., doi:10.1175/1520-0442(1995)008<2039:TLSSIW>2.0.CO;2.

Manabe S., 1969. Climate and the ocean circulation. Mon. Weather Rev., 97(11), 739-774, doi:10.1175/1520-0493(1969)097<0739:CATOC>2.3.CO;2.

Le Moigne P., Minvielle M., 2020. Surfex : une plateforme pour simuler les flux des surfaces océaniques et continentales. La Météorologie, 108, xx-xx.

Noilhan J., Lacarrère P., 1995. GCM grid-scale evaporation from mesoscale modeling. $J$. Clim., doi:10.1175/1520-0442(1995)008<0206:GGSEFM>2.0.CO;2.

Noilhan, J., Planton S., 1989. A simple parameterization of land surface processes for meteorological models. Mon. Weather Rev., 117(3), 536-549, doi:10.1175/15200493(1989)117<0536:aspols>2.0.co;2.

Parton W. J., Stewart J. W. B., Cole C. V., 1988. Dynamics of C, N, P and S in grassland soils: a model. Biogeochemistry, 5(1), 109-131, doi:10.1007/BF02180320.

Planton S., Mahfouf, J.-F., 2020. Une nouvelle description des surfaces continentales pour les modèles de Météo-France : les origines du schéma ISBA. La Météorologie, 108, xx-xx.

Planton S., Bopp L., Brun E., Cattiaux J., Chauvin F., Chevallier M., Ciais P., Douville H., Giraud G., Soubeyroux J.-M., Terray L., 2015. Evolution du climat depuis 1850. La Météorologie, 8(88), 48, doi:10.4267/2042/56361.

Séférian R., Nabat P., Michou M., Saint-Martin D., Voldoire A., Colin J., Decharme B., Delire C., Berthet S., Chevallier M., Sénési S., Franchisteguy L., Vial J., Mallet M., Joetzjer E., Geoffroy O., Guérémy J., Moine M., Msadek R., Ribes A., Rocher M., Roehrig R., Salasy-Mélia D., Sanchez E., Terray L., Valcke S., Waldman R., Aumont O., Bopp L., Deshayes J., Éthé C., Madec G., 2019. Evaluation of CNRM Earth System Model, CNRM-ESM2-1: Role of Earth System Processes in Present-Day and Future Climate. J. Adv. Model. Earth Syst., 2019MS001791, doi:10.1029/2019MS001791.

Sellers P. J., Mintz Y., Sud Y. C., Dalcher A.,1986.: A simple biosphere model (SiB) for use within general circulation models. J. Atmos. Sci., 43(6), 505-531, doi:10.1175/1520- 
0469(1986)043<0505:ASBMFU>2.0.CO;2.

Sellers P. J., Randall D. A., Collatz G. J., Berry J. A., Field C. B., Dazlich D. A., Zhang C., Bounoua L., 1996. A revised land surface parameterization (SiB2) for atmospheric GCMs. Part I: Model formulation. J. Clim., 9(4), 676-705, doi:10.1175/15200442(1996)009<0676:ARLSPF>2.0.CO;2. 


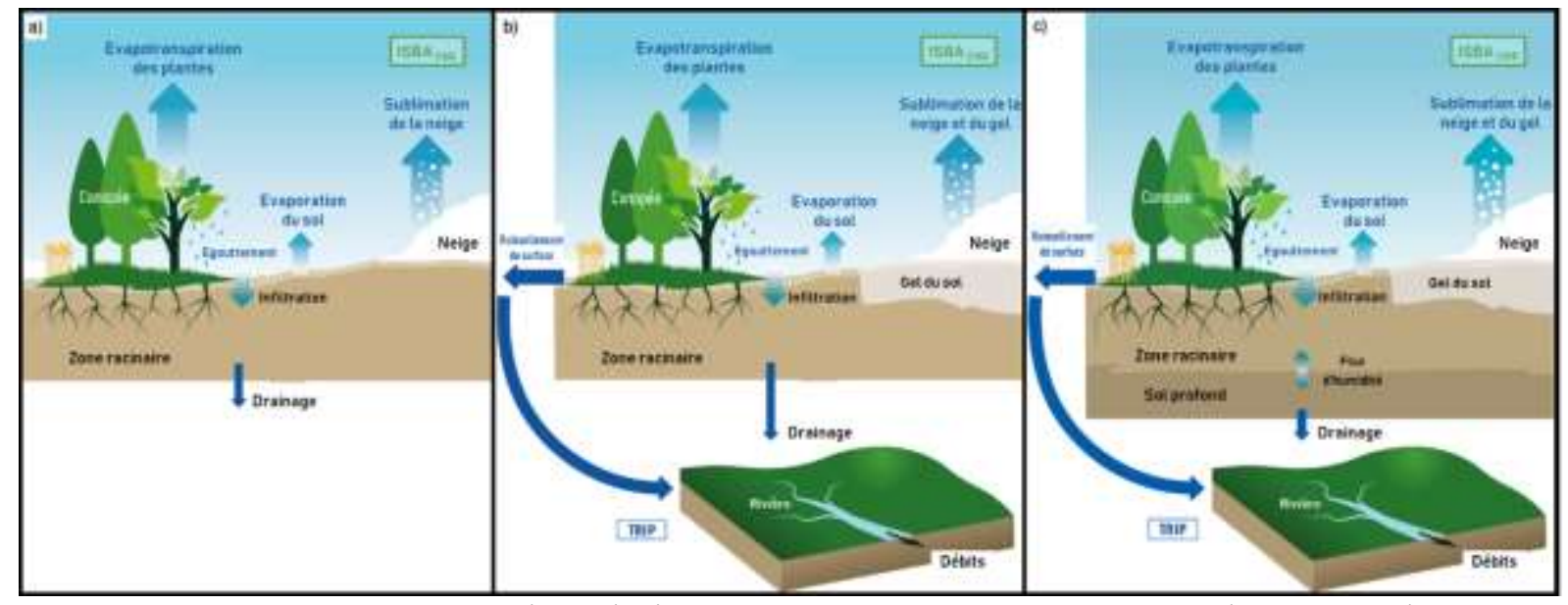

Figure 1 - Schémas montrant l'évolution des différentes configurations d'Isba a) à l'orée des années 2000 dans CNRM-CM2, b) puis couplé avec Trip au milieu des années 2000 dans CNRM-CM3, c) et enfin au début des années 2010 dans CNRM-CM5. D'après Decharme et Douville (2007). 

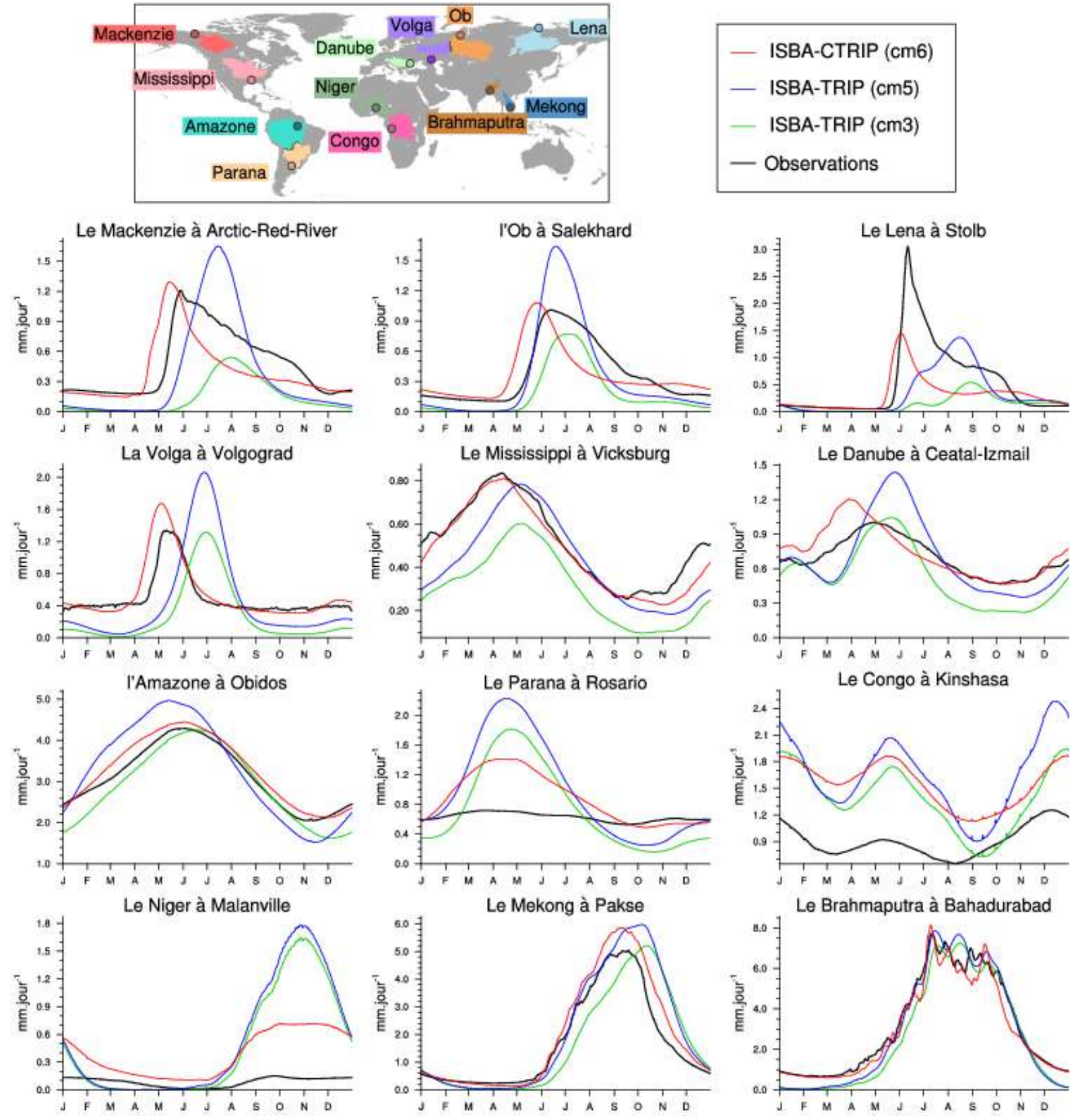

Figure 2 - Comparaison sur la période 1979-2010 entre les cycles annuels saisonniers moyens des débits quotidiens ( $\mathrm{mm}_{\text {.jour }}{ }^{-1}$ ) simulés et observés aux stations les plus proches de l'embouchure des principaux bassins du monde. Les débits simulés par les anciennes versions d'Isba utilisées dans CNRM-CM3 (vert) et CNRM-CM5 (bleu) ont été routés par Ctrip à $0.5^{\circ}$ de résolution avec une vitesse d'écoulement fixée à $0.5 \mathrm{~m} . \mathrm{s}^{-1}$. Le nouveau système Isba-Ctrip (rouge) utilisé dans CNRM-CM6 est aussi montré. Toutes ces versions ont été forcées avec les mêmes données atmosphériques tri-horaires à $1^{\circ}$ de résolution $(\sim 100$ $\mathrm{km}$ ) fournies par l'université de Princeton et issues des ré-analyses américaines combinées à des observations de température, de rayonnement et de précipitation. 


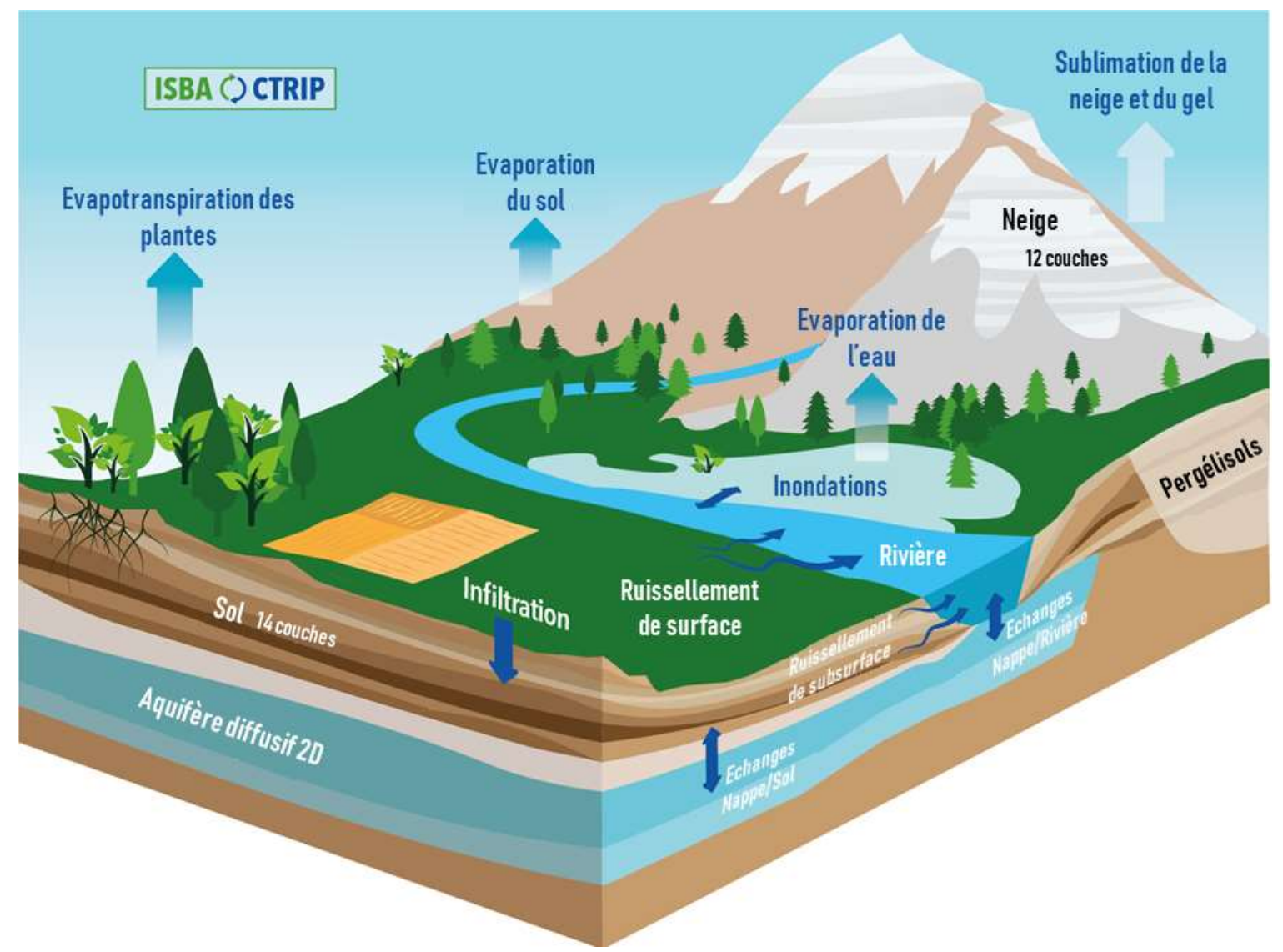

Figure 3 - Schéma du cycle continental de l'eau simulé par le système de modélisation IsbaCtrip utilisé actuellement à Météo-France pour des applications climatiques à l'échelle globale. D'après Decharme et al. (2019). 


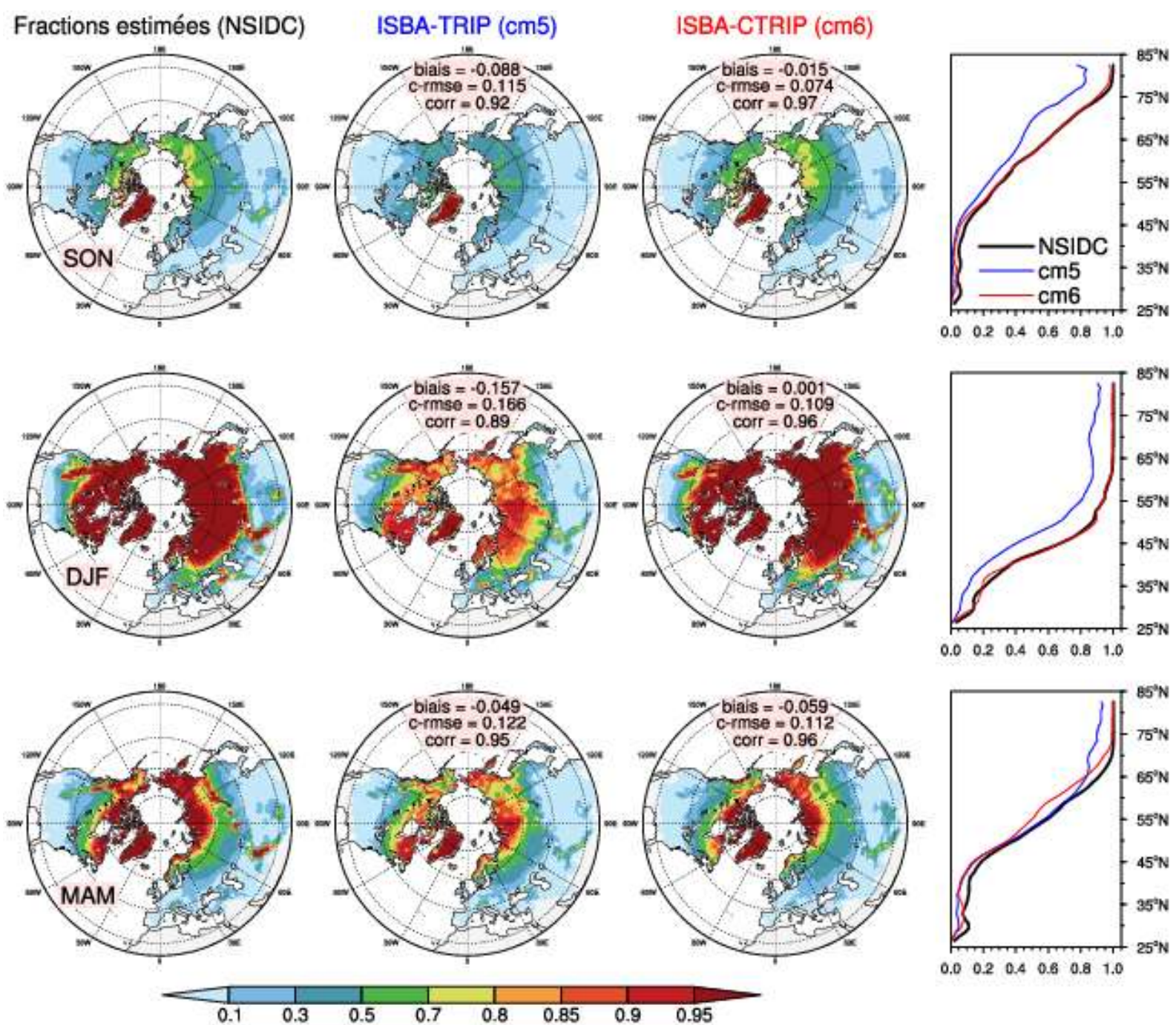

Figure 4 - Distribution et moyennes zonales dans l'hémisphère nord des étendues saisonnières moyennes de couverture de neige (exprimée en fraction de la maille) observées (noires) fournie par le NSIDC (National Snow and Ice Data Center) et simulées par l'ancien schéma de neige mono-couche d'Isba utilisée dans CNRM-CM5 (bleu) et le nouveau schéma de complexité intermédiaire d'Isba utilisé dans CNRM-CM6 (rouge) dans en automne (SON ; Septembre-Octobre-Novembre), en hiver (DJF ; Décembre-Janvier-Février) et au printemps (MAM ; Mars-Avril-Mai) sur la période 1979-2010. Les mêmes conditions expérimentales que pour la figure 2 ont été appliquées. Les scores proposés mesurent la cohérence spatiale entre modèles et observations en termes de biais moyen, d'erreur quadratique moyenne centrée (c-rmse) et decorrélation spatiale (corr). Plus les deux premiers scores sont proches de 0 et la corrélation de 1 , meilleure est la simulation. 
a) Estimations et simulations des limites du pergélisol et des profondeurs de sa couche active

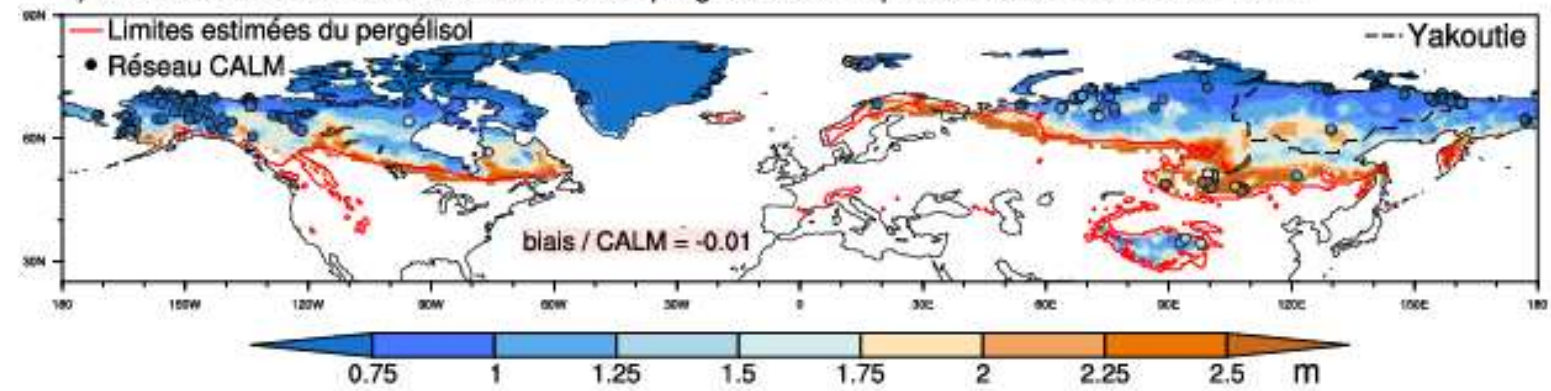

b) Profondeurs estimées en Yakoutie Profondeurs simulées
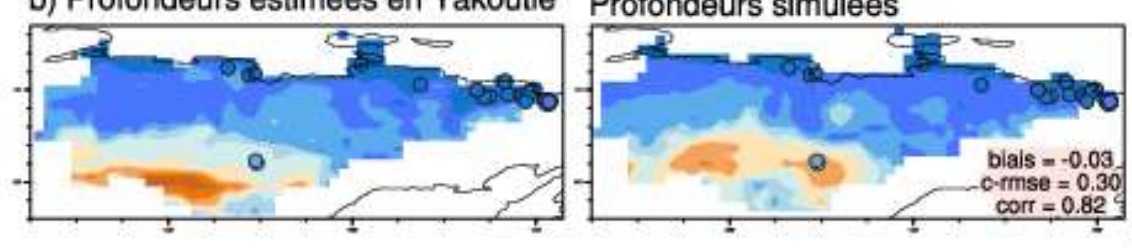

Moyennes zonales

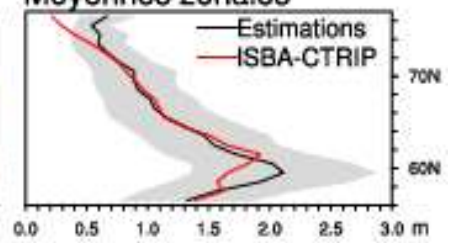

Figure 5 - Comparaison des caractéristiques observées et simulées du pergélisol dans l'hémisphère nord. (a) les profondeurs moyennes des couches actives simulées sur la période 1990-2010 par la version multi-couches d'Isba sont représentées en couleur et comparées à la limite estimée du pergélisol (trait rouge) fournie par le NSIDC et aux 209 observations insitu (cercles) du réseau Calm (Circumpolar Active Layer Monitoring). (b) Zoom sur la région de la Yakoutie où la profondeur de la couche active simulée par Isba est comparée à la fois aux réseau Calm et à des estimations russes spatialisées qui sont tous deux en bon accord. La cohérence spatiale entre ces estimations russes et la simulation est mesuré par des scores de biais moyen, d'erreur quadratique moyenne centrée (c-rmse) et de corrélation spatiale (corr). Les moyennes zonales de ces estimations sont données en noir avec leurs incertitudes en grisées alors qu'Isba-Ctrip apparait en rouge. 


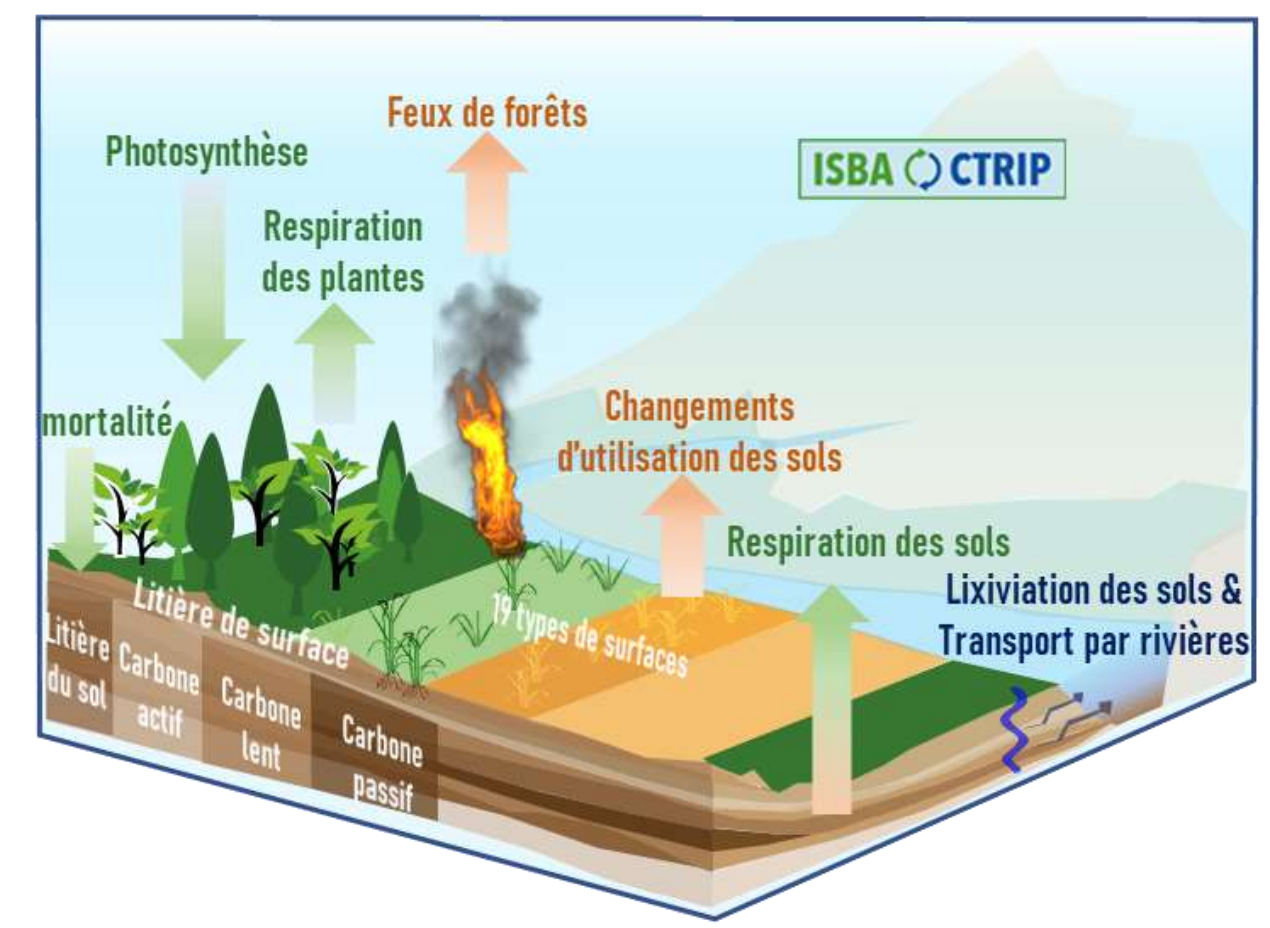

Figure 6 - Schéma du cycle continental du carbone simulé par la version «système Terre » d'Isba-Ctrip considérant19 types de surfaces (16 types de végétation, le sol nu, la glace et la roche). Les flux d'échanges de $\mathrm{CO}_{2}$ entre la surface et l'atmosphère sont : la photosynthèse (flux entrant), la respiration autotrophe (respiration des plantes, flux sortant), la respiration hétérotrophe des sols (décomposition de la matière organique, flux sortant) auxquels il faut ajouter les perturbations par les feux de forêts qui libèrent du $\mathrm{CO}_{2}$ et les changements d'utilisation des sols. Le carbone organique extrait par les mouvements de l'eau en surface et dans les sols (lixiviation) est transporté dans le réseau hydrographique jusqu'à l'océan. 

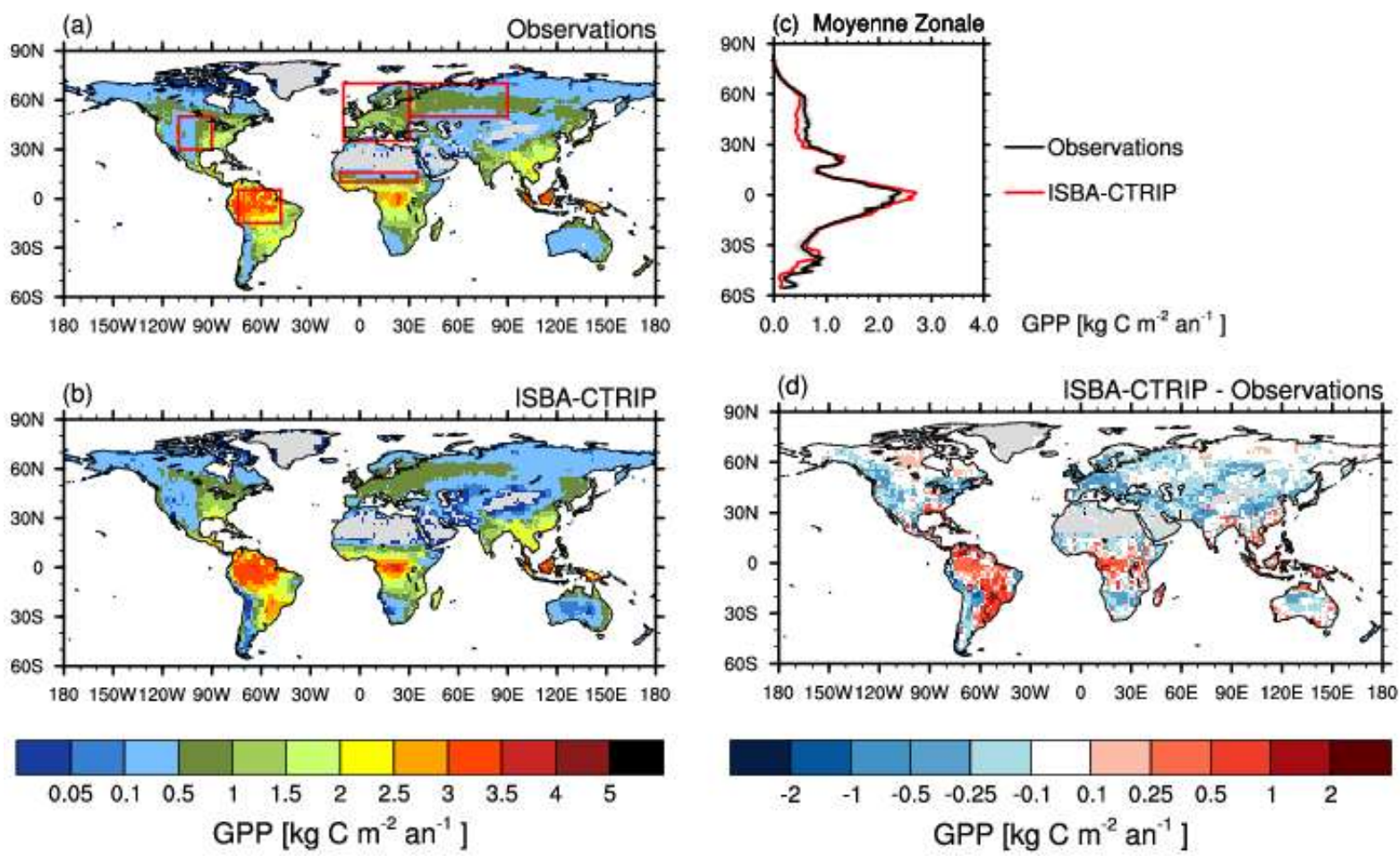

Figure 7 - Assimilation du carbone par photosynthèse (GPP ${ }^{5}$ en anglais). (a) GPP déduite d'observations in-situ spatialisées et fournies par le MPI (Max Planck Institute). Les rectangles rouges désignent les régions utilisées dans la figure 8. (b) GPP simulée avec Isba, forcé par des observations atmosphériques. (c) Moyennes zonales des GPP observées (noir) et simulées avec Isba (en rouge). (d) Différence de GPP entre simulation et observation. Le biais moyen est de $-0.031 \mathrm{kgC} \cdot \mathrm{m}^{-2} \cdot \mathrm{an}^{-1}$ et la corrélation spatiale de 0.93 .

\footnotetext{
${ }^{5}$ Gross primary production
} 

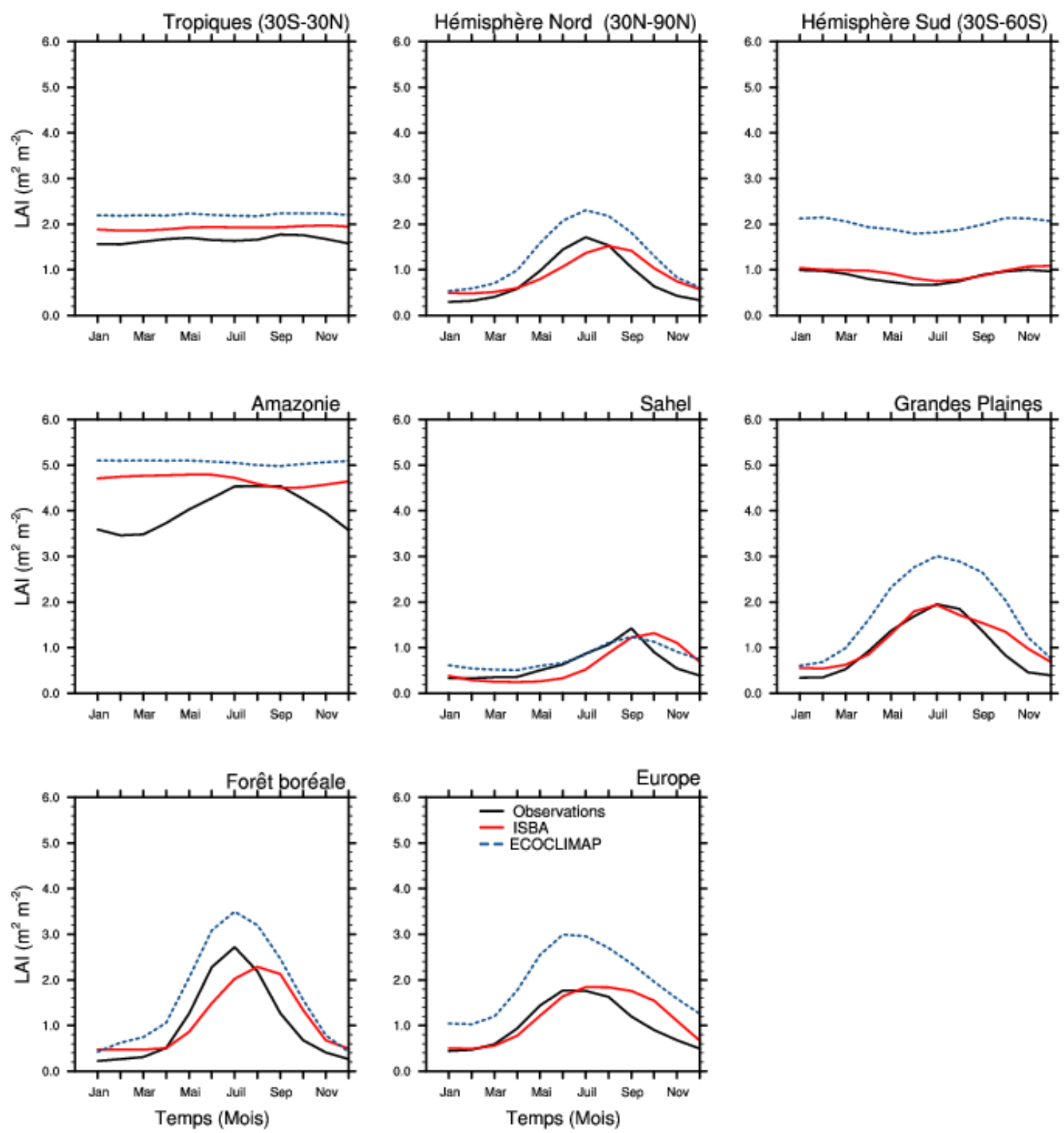

Figure 8 - Cycles saisonniers moyens de l'indice foliaire (LAI en anglais) observé par satellite en noir, et simulé avec Isba (en rouge) forcé par des observations atmosphériques. Les tirets noirs représentent la reconstruction de cet indice via la base de données physiographiques Ecoclimap. Les régions sont définies par les rectangles rouges de la figure $7 a$. 